\title{
LETTER
}

doi:10.1017/\$1041610212000415

\section{Informant Questionnaire on Cognitive Decline in the Elderly (IQCODE) completion on an acute care ward for the elderly: a brief study of informant characteristics}

Over recent years in the UK, emphasis has been placed on appropriate diagnosis and referral of patients with dementia. In guidelines published by the British Geriatrics Society (BGS) and Faculty of Old Age Psychiatrists consensus group (Forsyth et al., 2006), a cognitive screening algorithm was developed, which consists of initial screening for cognitive impairment with the Mini-Mental State Examination (MMSE) and CLOX1 (an executive clock drawing task). If the scores meet cut-off points indicated in the algorithm (MMSE $<24$ or CLOX1 <11), further assessments with the Confusion Assessment Method (CAM) and the Informant Questionnaire on Cognitive Decline in the Elderly (IQCODE) are applied with the aim to differentiate between delirium (CAM positive, IQCODE negative), delirium and chronic impairment (CAM positive, IQCODE positive), or chronic cognitive impairment (CAM negative, IQCODE positive).

Use of this algorithm on our acute older person's ward in a UK hospital demonstrated this to be feasible, with the time taken to complete most components being less than 15 minutes. The IQCODE, however, frequently required additional input. Completion and return rates from relatives on our wards have been found to be suboptimal, and members of the acute team have concerns about whether the closest informant was completing the IQCODE. The aim of our study was to determine characteristics of informants completing the IQCODE on an acute older person's ward.

Patients requiring IQCODE completion as per the BGS cognitive screening algorithm were identified. Patients who were palliative or too medically unwell to participate in initial cognitive screening were excluded. Patients' relatives/friends were approached during daytime visiting hours to complete a study questionnaire and an IQCODE. The study questionnaire consisted of questions designed to assess basic demographic information, relationship of the informant to the patient, and ease of use of the IQCODE.

During the study 57 patients were identified with algorithm scores on MMSE or CLOX1 indicating possible cognitive impairment and, therefore, requiring IQCODE completion. Of these, 23 patients $(40 \%)$ did not have an IQCODE completed due to absence of a relative on the ward during daytime hours. Thirty-four informants were approached. Twenty-four IQCODEs were completed and returned. This represented a completion rate of $42 \%$ from the total group requiring an IQCODE and $71 \%$ of those given the IQCODE. Of the patients with returned IQCODEs all but one was female, with a mean age of 87 years. One-third had a previous diagnosis of cognitive impairment. Twenty patients $(83 \%)$ had a positive IQCODE (score >3.43).

Most informants were adult children (13 informants, 54\%), and had a mean age of 65 years. Fifty percent (12 informants) considered themselves the primary caregiver, $88 \%$ (21 informants) reported being the best person to complete the IQCODE, and 46\% (11 informants) spent over 14 hours per week with the patient. Selfreported anxiety for completing the IQCODE was low (three informants) and 83\% (20 informants) felt confident in their responses.

Nine IQCODE questionnaires were not fully completed with between one and eight of the 16 questions omitted. In most cases, the informant indicated why the question concerned was not completed. Reasons included significant visual or other functional impairment interfering with specific tasks, that tasks had always been completed by a spouse, or that the patient had not performed a particular task often enough over 10 years.

In summary, our study shows that most informants of older inpatients are appropriate to complete the IQCODE, but uptake and response rates are suboptimal. Several questions are frequently omitted, which may reflect the older and frail nature of our inpatients. Our study results may also reflect a potential dementia diagnosis gap. This study involved a predominantly female patient cohort and, therefore, may not necessarily be fully generalized. IQCODE completion by a relative was suboptimal, with $40 \%$ of patients not having a relative or friend attend the ward during daytime hours when a member of the team would have been available. Contributing factors to this could be relatives working in the day (visiting after hours), the short average length of stay, and relatives living out of region. It is possible to perform the IQCODE over the phone with a relative; however, this is time consuming for the medical team, and possibly illtimed for a working relative. When IQCODEs are 
given to relatives to complete, response rates in this study were $71 \%$. Interestingly, all relatives except three asked if they could complete the IQCODE at home in their own time rather than on the ward. Relatives often forgot to return the IQCODE, or patients were discharged home prior to the relative returning to the ward with the completed IQCODE.

IQCODEs were fully completed for approximately $60 \%$ of patients in our study. We believe the lack of full completion is influenced by the older age and frailty of our patients, due to the acute inpatient being a different population to many of the earlier IQCODE study populations. Many of the published studies on the IQCODE have involved younger patients in the outpatient setting (Jorm, 2004). We have not found any published data on patient comorbidities in previous IQCODE studies but it is possible that the patients in our study have high rates of comorbid disease in comparison. Previous studies have varied in the number of items that users have allowed before counting the IQCODE as invalid.

In our patients who screened positive for cognitive impairment based on MMSE and/or CAM, 33\% had known cognitive impairment at the time of admission. Despite this, over $80 \%$ of patients had positive IQCODE scores. These results are consistent with a report published in 2007 by the National Audit Office which documented a significant diagnosis gap, with approximately one-third of patients receiving a formal diagnosis of dementia (National Audit
Office, 2007). Alternatively, the IQCODE could be overestimating rates of dementia in our inpatients.

In summary, there are significant issues of getting access to suitable informants and receiving completed IQCODEs in the acute inpatient hospital setting. Further study of the IQCODE in this population is necessary as some questions may lose validity in this old, frail patient group.

\section{Conflict of interest}

None.

\section{References}

Forsyth, D. et al. (2006). Delirious About Dementia. Available at: http//www.bgsnet.org.

Jorm, A. F. (2004). The Informant Questionnaire of Cognitive Decline in the Elderly (IQCODE): a review. International Psychogeriatrics, 16, 1-19.

National Audit Office (2007). Improving Services and Support for People with Dementia. London: NAO. Available at: http://www.nao.org.uk/publications/0607/dementia_ services_and_support.aspx.

\footnotetext{
K. BLOOMFIELD ${ }^{1}$ AND N. JOHN ${ }^{2}$

${ }^{1}$ Freemasons' Department of Geriatric Medicine, University of Auckland and Waitemata District Health Board, Auckland, New Zealand Email: katherine.bloomfield@waitematadhb.govt.nz ${ }^{2}$ Mater Private Hospital Redland, Cleveland, Queensland, Australia
} 